\title{
MILP model for integrated line balancing and model sequencing problems for Mixed-Model Two-Sided Assembly Line
}

\author{
Nur Intan Liyana Mohd Azmi a, Zaitul Marlizawati Zainuddin b,", Rashidah Ahmad c, \\ ${ }^{a, b}$ Department of Mathematical Sciences, Faculty of Science, Universiti Teknologi Malaysia, 81300 Skudai, Johor, Malaysia \\ c UTM Centre for Industrial and Applied Mathematics (UTM-CIAM), Faculty of Science, Universiti Teknologi Malaysia, 81300 Skudai, Johor, \\ Malaysia \\ *Corresponding author: rashidahahmad@utm.my, zmarlizawati@utm.my
}

\section{Article history}

Received 6 November 2017

Accepted 8 November 2017

\begin{abstract}
This research explores two interrelated problems in Mixed-Model Two-Sided Assembly Line (MMTSAL), which are line balancing and model sequencing. These two problems are solved simultaneously using Mixed Integer Linear Programming (MILP) with the objectives of minimizing total utility work and idle time by considering various practical constraints. The problem is analyzed using small-size to large-sized test cases using General Algebraic Modelling System (GAMS) with the solver CPLEX. Experimental results indicate that integrating the problems help to minimize the proposed objective function. Also, it is found that the feasible solution for model sequence with the assignment of tasks to assembly line is optimal.
\end{abstract}

Keywords: Mixed-model two-sided assembly line, model sequencing, line balancing, simultaneous

line balancing and mixed-model sequencing, mixed integer linear programming

\section{INTRODUCTION}

Mixed-model two-sided assembly lines (MMTSAL) are designed for producing high volume intermixed product's model. Large industries such as automotive industry adapt these line to satisfy the market demand. Along the line, successional mated stations consist of left and right workstations are placed facing each other and are connected by mechanical transportation mechanism such as steadily moving conveyer belt. The product units to be assembled move along the line and visit each station at a fixed rate of time span called cycle time. Within the limited length of workstation (work area), operators are placed which each is responsible for each workstation to perform a non-overlapping task without interfering with one another [1]. To increase the productivity of MMTSAL, it is necessary for the operators to perform their operations from both sides of the line in parallel. The tasks to be performed in MMTSAL have restrictions on the operation directions. That is, some tasks may be performed on a specific side of the line whether it is left or right side, while others may be performed on either side of the line [2]. Therefore, the tasks are classified into three types which are left-side tasks (L), right-side tasks (R) and eitherside tasks (E).

MMTSAL consists of two different problems that are line balancing problem and model sequencing problem. Line balancing is the problem of assigning tasks to workstations without violating the precedence constraint and other restrictions. While model sequencing is the problem of determining a production sequence of models which means various and different models of the same product are intermixed to be assembled on the same line. At the beginning stage of this studies of line balancing and model sequencing, many researches have worked on it separately.

In the context of line balancing problem of the two-sided assembly line (TSAL), comprehensive studies with various objective functions have been done in [2-8]. Lee et al. [3] studied TSAL to maximize work relatedness and slackness which they assigned a group of tasks at a time rather than a unit task. Kim et al. [4] was the first proposed that MILP model considering sequence dependent finishing time of tasks. Then, Ozcan and Toklu [2] extended the study in [4] and introducing specific additional constraints in the MILP such as zoning constraints, positional constraints and synchronism constraints. Chutima and Chimklai [5] considered the negative knowledge to solve the multiobjective TSAL. Purnomo et al. [6] considered assignment restrictions in which the constraints used are the same as in [2] except that by adding two more constraints which are distance and resource constraints. Khorasanian et al. [7] considered the relationship between tasks by introducing three performance criteria in simulated annealing method.

For model sequencing problem, many researchers studied mixedmodel assembly line (MMAL) of traditional straight line and most of them focused on minimizing total utility work such as Yano and Rachamadugu [8]. The study by Fattahi and Salehi [9] focused on minimizing the cost of total utility work and idle time with variable launching interval. They also evaluated on fixed launching interval and compared it with the variable solution. Another study by Sarker and Pan [10] also solved the model sequencing problem as in [9] with fixed launching interval. However they focused on designing MMAL of the problems for open and closed station.

A specific review on MMAL of model sequencing problems has been presented by Boysen et al. [11]. Work overload or utility work is defined as the amount of work that is not completed within the given length of workstation and is typically handled through the use of utility workers who assist the regular workers during work overload. According to the study in [11], this objective contributes to reducing not only labor cost but the risk of stopping the conveyor and the required line length. Apart from the studies in [8-10], only Chutima and Naruemitwong [1] studied MMTSAL for solving the multiobjective of sequencing problems.

Line balancing and model sequencing problems have also been studied in a hierarchical manner which solves one problem first and 
then the other under the constraint of the first solution such as Thomopoulos [12], Dar-El and Nadivi [13] and Sawik [14]. According to Kim and Kim [15], solving both problems hierarchically have a limitation in exploring the solution space. Both problems are very tightly interwoven with each other where the optimality of model sequences depends on the results of the task assignment. Therefore, researchers started to realize that line balancing and model sequencing problems are somehow interrelated to each other and this problem has been paid great attention to be solved simultaneously.

Kim and Kim [15] was the first presented the integrated approaches of MMAL with the objective function of minimizing utility work and to solve it simultaneously using the co-evolutionary algorithm. Meanwhile, Kara [16] proposed simultaneous solution for U-shape assembly line and minimizing the absolute deviation of workload. Then, Mosadegh et al. [17] proposed first MILP model of simultaneous line balancing and model sequencing for MMAL. In this study, they minimized the total utility work and presented the exact solution of the MILP together with solution of simulated annealing and coevolutionary genetic algorithm.

In this paper, a mixed-model two-sided assembly line (MMTSAL) with sequence dependence finishing time is considered to solve simultaneously the integration of line balancing and model sequencing problems. The objective functions are to minimize the total utility works and idle time. To the best of our knowledge, the MILP model of MMTSAL for minimizing aforementioned objectives in this research is the first in the literature. The MILP is solved using General Algebraic Modelling System (GAMS) with the solver CPLEX.

\section{PROBLEM DESCRIPTION}

In MMTSAL, sets of similar product models are assembled in any model sequence and model mix. Each model has its own set of tasks and some of the tasks are the common basis that allows the tasks to be combined in one precedence diagram as in Figure 1. From this combined precedence diagram, a model sequence needs to be generated and tasks will be assigned to workstations in such a way that all constraints are satisfied.

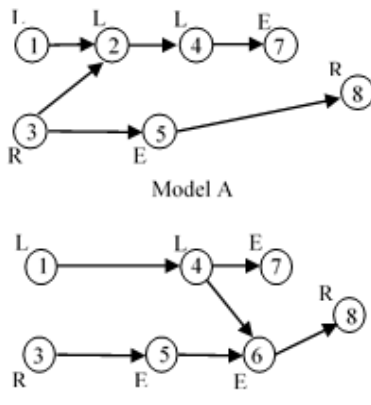

Model C
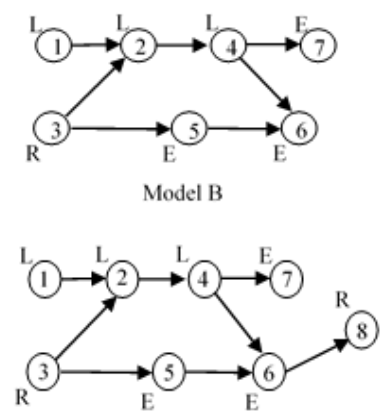

Combine Precedence Diagram
Fig. 1 Precedence diagram

Precedence and line constraint need to be satisfied when assigning tasks to workstation. In MMTSAL, interference phenomenon also known as sequence-dependent finish time is one of the problems that may occur during assigning tasks to workstation. It happens because some workstations need to wait for a predecessor task to be completed at the opposite side of the line before starting to operate a new task. Figure 2 shows the example of interference phenomenon in which task $i$ and task $j$ are two immediate predecessors of task $k$. Task $i$ is assigned to left side of station 1 while task $j$ is assigned to the right side of station 2 . Since the processing time for task $j$ is longer than task $i$, the operator at the left side of station 1 needs to wait for the operator at the right side of station 2 to complete his work before continuing to work on task $k$. The waiting time by the operator at station 1 before starting task $k$ is known as idle time and it is unavoidable. This problem is called interference phenomenon.

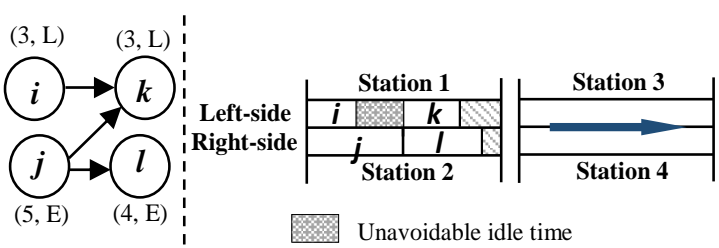

Fig. 2 Interference phenomenon in MMTSAL

In order to solve models sequencing problem, model mix in a production cycle or also known as Minimum Part Set (MPS) is manufactured. MPS is represented by $d_{m}=D_{m} / h$ where $d_{m}=\left(d_{1}, d_{2}, \ldots, d_{m}\right)$ is the demand vector of each model and $D_{m}=\left(D_{1}, D_{2}, \ldots, D_{m}\right)$ is the planning horizon or demands. While $h$ is the greatest common divisor that will be obtained from the demands. To explain the problem, assume three models namely A, B and C with their demands of 200, 100, and 200 respectively. Therefore from the demands given, $h$ is 100 and the demand vector becomes $d_{m}=(2,1,2)$.

Hence, the model sequence can be obtained as ACBAC. However, the model sequence obtained in the MPS cycle must be numbered to distinguish the task assignment. This is because the task assignment might differ even for the same models. For example, producing car model A might differ in task sequence since car A has options of manual and auto transmission. Hence, the model sequence can be numbered as $\mathrm{A}_{1} \mathrm{C}_{1} \mathrm{~B}_{1} \mathrm{~A}_{2} \mathrm{C}_{2}$. The sequence and the assignment of tasks is illustrated in Figure 3.

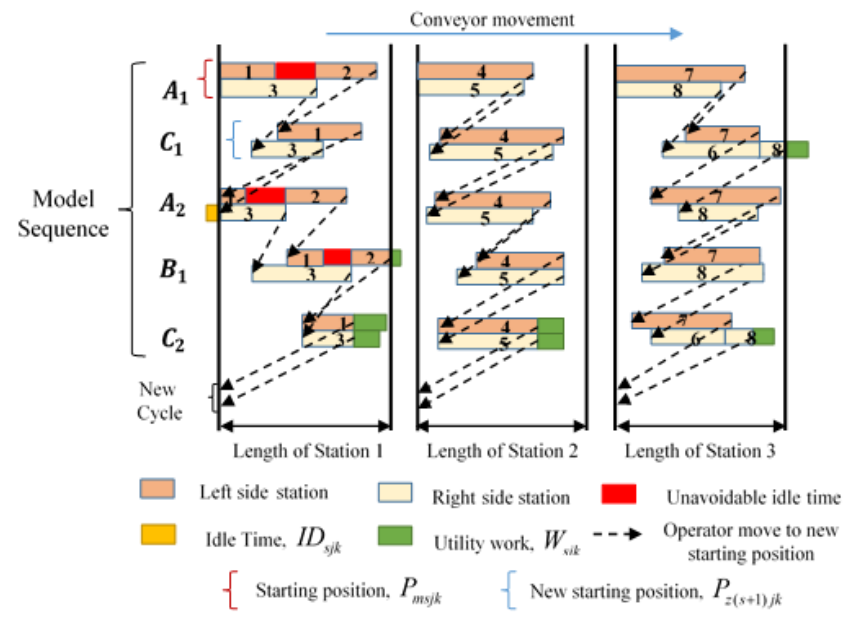

Fig. 3 An example of model sequence, task assignment, required utility works and idle time in MMTSAL. The data is given in Fig. 1.

Assume that there are three workstations, the launching rate of work-pieces is fixed and the conveyor belt is constant. Feasible tasks sequence is obtained from the precedence diagram in Figure 1 and the model sequence obtained previously is $\mathrm{A} 1 \mathrm{C} 1 \mathrm{~B} 1 \mathrm{~A} 2 \mathrm{C} 2$. Operators begin operating first task from left and move downstream within their allowable work area. If the tasks are not completed by the time it reaches the boundary, operators need to return to their starting position to start operating next task and additional operator or utility worker helps to complete the unfinished task. The operators moving time from end boundary to the next starting position is ignored. If the next task has not yet entered the operator's allowable work area, they need to wait for it and this waiting time is known as idle time. After finishing one cycle, the operator must be at the beginning of the station which causes uncompleted tasks and it is taken into account as utility work.

Both problems of model sequencing and line balancing will be solved simultaneously. The following assumptions are considered to model MMTSAL.

(i) Product models with similar production characteristics are produced on the same two-sided assembly line. 
(ii) Some tasks may be required to be performed at one-sided of the line, while others may be performed at either side of the line.

(iii) The combine precedence diagram concept is employed where each of the precedence diagrams of each model is known.

(iv) Minimum Part Set (MPS) is used.

(v) A completion time may be differed from one model to another and can be equal to zero.

(vi) Task time is deterministic.

(vii) Tasks are performed by operators in parallel at both sides of the line.

(viii)Travel time of operators is ignored.

(ix) The launching rate of each model is fixed and the speed of the conveyor is constant.

(x) All workstations are a closed type and the number of workstations is predetermined.

(xi) The uncompleted tasks are passed to the utility worker.

\section{MATHEMATICAL FORMULATION}

The modeling developed in this paper is a modified model originated from Mosadegh et al. [17]. The characteristic of MMTSAL is added to the model. The following notation is used to develop the model.

\section{Indices}

$i, h, p, r \quad$ Task

$j, g \quad$ Mate station

$m \quad$ Product model

$s \quad$ Sequence

$(j, k) \quad$ Station of mate-station $j$ and its operation direction is $k$

$k, f \quad$ Side of the line

$k, f= \begin{cases}1 & \text { indicates a left-sidestation } \\ 2 & \text { indicates a right-side station }\end{cases}$

\section{Parameters}

I Set of task; $I=\{1,2, . ., i, . . n t\}$

$S \quad$ Set of model sequence with $S=\{1,2, . ., i, . . n s\}$

$J \quad$ Set of mate stations $J=\{1,2, . ., i, . . n m s\}$

$M \quad$ Set of product models with $M=\{1,2, . ., i, . . n p m\}$

$A_{L} \quad$ Set of tasks which should be performed at left-side stations; $A_{L} \subset i$

$A_{R} \quad$ Set of tasks which should be performed at right-side stations; $A_{R} \subset i$

$A_{E} \quad$ Set of tasks which may be performed at either-side of a stations; $A_{E} \subset i$

$P(i) \quad$ Set of immediate predecessor of task $i$

$P_{a}(i) \quad$ Set of all predecessor of task $i$

$P_{0} \quad$ Set of task that have no immediate predecessor $P_{0}=\{I \in i \mid P(i)=\varnothing\}$

$S(i) \quad$ Set of immediate successor of task $i$

$S_{a}(i) \quad$ Set of all successor of task $i$

$t_{i m} \quad$ Completion time of task $i$ for model $m$

$v$ Speed of conveyor movement

$\gamma \quad$ Launching rate of each model

$d_{m} \quad$ Demand of model $m$ in the MPS cycle

$L_{j} \quad$ Length of mate-station $j$

$\psi \quad$ A very large positive number
$C(i) \quad$ Set of tasks whose operation directions are opposite to operation direction of task $i ; C(i)= \begin{cases}A_{L} & \text { if } i \in A_{R} \\ A_{R} & \text { if } i \in A_{L} \\ \varnothing & \text { if } i \in A_{E}\end{cases}$

$K(i) \quad$ Set of indicating the preferred operation direction of task $i$;

$$
K(i)= \begin{cases}1 & \text { if } i \in A_{R} \\ 2 & \text { if } i \in A_{L} \\ \{1,2\} & \text { if } i \in A_{E}\end{cases}
$$

\section{Decision Variables}

$X_{i j k} \quad 1$, if task $i$ of model $m$ is assigned to station $(j, k) ; 0$, otherwise

$Y_{m s} \quad 1$, if model $m$ is performed at sequence $s ; 0$, otherwise

$W_{s j k} \quad$ Utility work that occurred at sequence $s$ of station $(j, k)$ ; 0 , otherwise

$E P_{j k} \quad$ Ending position of operator for last model at station $(j, k)$

$I D_{s j k} \quad$ Idle time that occurred at sequence $s$ of station $(j, k)$

$t_{i m}^{f} \quad$ Finish time of task $i$ of model $m$

$P_{m s j k} \quad$ Start position of operator for model $m$ of sequence $s$ at station $(j, k)$

\section{Indicator Variables}

$Z_{i p} \quad 1$, if task $i$ is assigned earlier than task $p$ in the same station; 0 , otherwise.

The mathematical model of MMTSAL for minimizing total utility works and idle time is as follows:

$$
\text { Minimize } Z=\sum_{j \in J} \sum_{k \in K}\left(\sum_{s \in S}\left(W_{s j k}+I D_{s j k}\right)+E P_{j k}\right)
$$

Subject to:

$P_{m s j k}+\left(\sum_{i \in I} t_{i m} X_{i j k}\right) v_{c}-W_{s j k} \leq L_{j} \quad \forall j \in J, k \in K(i), \forall m \in M, \forall s \in S$

$P_{m s j k}+\left(\sum_{i \in I} t_{i m} X_{i j k}\right) v_{c}-W_{s j k}+I D_{(s+1) j k}+\left(Y_{m s}+Y_{z(s+1)}-2\right) \psi-\gamma v \leq P_{z(s+1) j k}$

$\forall j \in J, k \in K(i), \forall m \in M, \forall s=1, . ., S-1, z \in M$

$P_{m s j k}+\left(\sum_{i \in I} t_{i m} X_{i j k}\right) v_{c}-W_{s j k}+\left(Y_{m s}-1\right) \psi-\gamma v \leq E P_{j k}$

$\forall j \in J, k \in K(i), \forall m \in M, \forall s \in S$

$\sum_{j \in J} L_{j}-\left[P_{m(s-1) j k}+\left(\sum_{i \in I} t_{i m} X_{i j k}\right) v_{c}-W_{(s-1) j k}-\gamma v\right]+\left(Y_{m s}-1\right) \psi \leq I D_{s j k}$

$\forall j \in J, k \in K(i), \forall m \in M, \forall s=2, . ., S-1$

The objective in (1) is to minimize the total utility work and idle time for one cycle. In constraint (2), the utility work of each sequence at each side of stations is computed. Constraint (3) is related to the new starting position of operator after finishing each model. Constraint (4) calculates the value of ending position of uncompleted task of the last model in a cycle which also act as utility work. The idle time is computed in constraint (5).

$\begin{array}{ll}P_{m s j k} \leq \psi Y_{m s} & \forall j \in J, \quad k \in K(i), \forall m \in M, \forall s \in S \\ \sum_{s \in S} Y_{m s}=d_{m} & \forall m \in M \\ \sum_{m \in M} Y_{m s}=1 & \forall s \in S \\ \sum_{j \in J} \sum_{k \in K} X_{i j k}=1 & \forall i \in I\end{array}$




$\begin{array}{ll}\sum_{j} X_{i j 1}=1 & \forall i \in A_{L} \\ \sum_{j} X_{i j 2}=1 & \forall i \in A_{R}\end{array}$

Constraint (6) connects the value of $P_{m s j k}$ to the value of $Y_{m s}$.

Constraint (7) guarantees that the demand for each model in MPS cycle is satisfied. Constraint (8) ensures that exactly one model is assigned to each position in a sequence. Constraint (9) is the occurrence constraint which a task is only assigned to one workstation. Constraint (10)-(11) enforce the tasks with specific operation direction to be assigned to the appropriate side of the station.

$\sum_{g \in J} \sum_{k \in k(h)} g X_{h g k} \leq \sum_{j \in J} \sum_{k \in k(i)} g X_{i g k} \forall i \in I-P_{0}, h \in P(i)$

$t_{i m}^{f}-t_{h m}^{f}+\psi\left(1-\sum_{k \in K(h)} X_{h j k}\right)+\psi\left(1-\sum_{k \in K(i)} X_{i j k}\right) \geq t_{i m}$

$\forall i \in I-P_{0}, \forall j \in J, h \in P(i)$

$t_{p m}^{f}-t_{i m}^{f}+\psi\left(1-X_{p j k}\right)+\psi\left(1-X_{i j k}\right)+\psi\left(1-Z_{i p}\right) \geq t_{p m}$

$\forall i \in I-P_{0}, \forall m \in M, \forall j \in J$,

$p \in\left\{r \mid r \in I-\left(P_{a}(i) \cup S_{a}(i) \cup C(i)\right)\right.$ and $\left.i<r\right\}$,

$k \in K(i) \cap K(p)$

$t_{i m}^{f}-t_{p m}^{f}+\psi\left(1-X_{p j k}\right)+\psi\left(1-X_{i j k}\right)+\psi Z_{i p} \geq t_{i m}$

$\forall i \in I-P_{0}, \forall m \in M, \forall j \in J$,

$p \in\left\{r \mid r \in I-\left(P_{a}(i) \cup S_{a}(i) \cup C(i)\right)\right.$ and $\left.i<r\right\}$,

$k \in K(i) \cap K(p)$

Constraint (12) is the precedence constraint which means that task can only be assigned to station if all of its predecessors are finished. Constraint (13)-(15) are introduced by Kim et al. [4] which are related to the sequence dependence finishing time. Constraint (13) is applied to a pair task such that task $h$ is the immediate predecessor of task $i$, then both tasks are assigned to the same station $j$. When this hold, the constraint is reduced to $t_{i m}^{f}-t_{h m}^{f} \geq t_{i m}$. This represents that, operator can start working on task $i$ immediately after task $h$ is finished. Constraint (14) and (15) are applied to two tasks that do not have precedence relations such that both tasks $i$ and $p$ are assigned to the same station $j$. If task $i$ is assigned earlier than task $p$ in the same station, then constraint (14) is active and reduced to $t_{p m}^{f}-t_{i m}^{f} \geq t_{p m}$. Otherwise, if task $p$ is assigned earlier than task $i$ in the same station, then constraint (15) becomes $t_{i m}^{f}-t_{p m}^{f} \geq t_{i m}$.

$$
\begin{array}{ll}
t_{i m}^{f} \geq t_{i m} & \forall i \in I, \forall m \in M \\
X_{i j k}=0,1 & \forall i \in I, \forall j \in J, k \in K(i) \\
Y_{m s}=0,1 & \forall m \in M, \forall s \in S \\
Z_{i p}=0,1 & \forall i \in I, \\
p \in\left\{r \mid r \in I-\left(P_{a}(i) \cup S_{a}(i) \cup C(i)\right) \text { and } i<r\right\}
\end{array}
$$

Constraint (16) ensures the finishing time of task $i$ for product model $m$ must be greater than or equal to the completion time of task $i$ for model $m$. Constraint (17)-(19) are the integrality constraints which restate the definition of variables.

\section{EXPERIMENTAL RESULT}

Due to the limitation of benchmark data of MMTSAL, five test problem of TSAL are used. Small size test problems, P12, P16 and P24 can be found in [4] and the large size test problem of P65 and P205 can be found in [3]. Number of tasks, number of station and number of model used are shown in Table 1. Since, the data for processing time in TSAL are only for one model, we added the processing time for mixed-model where the processing time is randomly generated between the values of 0 to 10 . The data used in this paper need to be analyzed first in order to generate a new data set that satisfying the conditions in the model. All of these data are analyzed using C++ of MS Visual Studio 2017 before they can be used to solve the MILP model. Then, the MILP is solved using General Algebraic Modelling System (GAMS) with the solver CPLEX on PC Intel (R) Core (TM) i7-3770, $3.40 \mathrm{GHz}$ processor and $8 \mathrm{~GB}$ memory.

Table 1 Data of five test problems.

\begin{tabular}{cccc}
\hline Test Problems & No. of tasks & $\begin{array}{c}\text { No. of } \\
\text { station }\end{array}$ & $\begin{array}{c}\text { No. of } \\
\text { model }\end{array}$ \\
\hline P12 & 12 & 3 & 3 \\
P16 & 16 & 3 & 3 \\
P24 & 24 & 4 & 4 \\
P65 & 65 & 14 & 4 \\
P205 & 205 & 43 & 4 \\
\hline
\end{tabular}

The experiments are conducted to investigate the performance of proposed method. The exact solutions obtained are shown in Table 2. It is shown that only small-size test problems give a feasible solution of the model. The optimal solutions for total utility work and idle time are obtained as 5.5, 24.3 and 37.1 for $\mathrm{P} 12, \mathrm{P} 16$ and $\mathrm{P} 24$ respectively. While, the optimal model sequence are obtained as $\mathrm{C}_{1} \mathrm{~A}_{1} \mathrm{C}_{2} \mathrm{~A}_{2} \mathrm{~B}_{1}, \mathrm{~A}_{1} \mathrm{C}_{1} \mathrm{~B}_{1} \mathrm{~A}_{2} \mathrm{C}_{2}$ and $\mathrm{C}_{1} \mathrm{~A}_{1} \mathrm{~A}_{2} \mathrm{~B}_{1} \mathrm{C}_{2} \mathrm{D}_{1}$ for $\mathrm{P} 12, \mathrm{P} 16$ and $\mathrm{P} 24$ respectively. However, as can be seen in Table 1, there are no solutions exist for test problem P65 and P205 because the execution was interrupted at CPU time 1000.36 second. The interruption happened because the running time reached its limit.

Table 2 Result of executing on five test problems

\begin{tabular}{ccccc}
\hline $\begin{array}{c}\text { Test } \\
\text { Problems }\end{array}$ & MPS & Objective & $\begin{array}{c}\text { CPU } \\
\text { time }\end{array}$ & $\begin{array}{c}\text { Optimal } \\
\text { Model } \\
\text { Sequence }\end{array}$ \\
\hline P12 & $(2,1,2)$ & 5.5 & 0.016 & $\mathrm{C}_{1} \mathrm{~A}_{1} \mathrm{C}_{2} \mathrm{~A}_{2} \mathrm{~B}_{1}$ \\
P16 & $(2,1,2)$ & 24.3 & 19.000 & $\mathrm{~A}_{1} \mathrm{C}_{1} \mathrm{~B}_{1} \mathrm{~A}_{2} \mathrm{C}_{2}$ \\
P24 & $(2,1,2,1)$ & 37.1 & 1000.02 & $\mathrm{C}_{1} \mathrm{~A}_{1} \mathrm{~A}_{2} \mathrm{~B}_{1} \mathrm{C}_{2} \mathrm{D}_{1}$ \\
P65 & $(2,3,2,1)$ & - & 1000.36 & - \\
P205 & $(1,3,2,2)$ & - & 1000.36 & - \\
\hline
\end{tabular}

\section{CONCLUSION}

This paper deals with solving simultaneously the integration problems of line balancing and model sequencing. A MILP on mixedmodel two-sided assembly line (MMTSAL) is presented. The mathematical modeling developed considers the objective function of minimizing the total utility work and idle time with the sequencedependence finishing time as the constraint. Another constraint that represents the characteristics of two-sided line and model sequencing is also been used. The result obtained showed that the model is feasible and the solution is optimal only for small-size test problems. The line balancing and model sequencing problems in MMTSAL is proven NPhard problems the computational time tends to be very long when the number of data size increase. Hence, it is recommended for future 
research that is to solve the MMTSAL by using fast and effective algorithms. Also, this research can be enriched with other assumptions related to MMTSAL such as assignment restriction, zoning constraint, positional constraint or resource constraint.

\section{ACKNOWLEDGEMENT}

This work was financially supported by Universiti Teknologi Malaysia (UTM), Ministry of High Education (MOHE) and STEM Grant with vote no. A. J091002.5600.07397, Research University Grant Scheme, are gratefully acknowledged.

\section{REFERENCES}

[1] Chutima, P. and Naruemitwong, W. A Pareto Biogeography-based Optimisation for Multi-objective Two-sided Assembly line Sequencing Problems with Learning Effect. Computers \& Industrial Engineering. 2014. 69: 89-104.

[2] Ozcan, U. and Toklu, B. Balancing of Mixed-model Two-sided Assembly Lines. Computers \& Industrial Engineering. 2009. 69: 217-227.

[3] Lee, T. O., Kim, Y. and Kim, Y. K. Two-sided Assembly Line Balancing to Maximize Work Relatedness and Slackness. Computers \& Industrial Engineering. 2001. 40: 273-292.

[4] Kim, Y. K., Song, W. S. and Kim, J. H. A mathematical Model and a Genetic Algorithm for Two-sided Assembly Line Balancing. Computers \& Operations Research. 2009. 36:853-865.

[5] Chutima, P. and Chimklai, P. Multi-objective Two-sided Mixed-model Assembly Line Balancing using Particle Swarm Optimisation with Negative Knowledge. Computers \& Industrial Engineering. 2012. 62: 39 55 .
[6] Purnomo, H. D., Wee, H. M. and Rau, H. Two-sided Assembly Lines Balancing with Assignment Restrictions. Mathematical and Computer Modelling. 2013. 57:189-199.

[7] Khorasanian, D., Hejazi, S. R. and Moslehi, G. Two-sided Assembly Line Balancing Considering the Relationships between Tasks. Computers \& Industrial Engineering. 2013. 66: 1096-1105.

[8] Yano, C. A. and Rachamadugu, R. Sequencing to Minimize Work Overload in Assembly Lines with Product Options. Management Science. 1991. 37(5):572-586.

[9] Fattahi, P. and Salehi, M. Sequencing the Mixed-model Assembly Line to Minimize the Total Utility and Idle Costs with Variable Launching Interval. International Journal Advance Technology. 2009. 45: 987-998.

[10] Sarker, B. R., and Pan, H. Designing a Mixed-Model Assembly Line to Minimize the Costs of Idle and Utility Times. Computers \& Industrial Engineering. 1998. 34(3): 609-628

[11] Boysen, N., Fliedner, M. and Scholl, A. Sequencing Mixed-model Assembly Lines: Survey, Classification and Model Critique. European Journal of Operational Research. 2009. 192: 349-373.

[12] Thomopoulos, N. T. Line Balancing-sequencing for Mixed Model Assembly. Management Science. 1967. 14: 59-75.

[13] Dar-El, E. M. and Nadivi, A. A Mixed Model Sequencing Application. International Journal of Production Research. 1981. 19: 69-84.

[14] Sawik, T. Monolithic vs. Hierarchical Balancing and Scheduling of a Flexible Assembly Line. European Journal of Operational Research. 2002. 143: 115-124.

[15] Kim, Y. K. and Kim, J. Y. A Coevolutionary Algorithm for Balancing and Sequencing in Mixed Model Assembly Lines. Applied Intelligence. 2000. 13: $247-258$.

[16] Kara, Y. Line Balancing and Model Sequencing to Reduce Work Overload in Mixed-Model U-line Production Environments. Engineering Optimization. 2008. 40(7): 669-684.

[17] Mosadegh, H., Zandieh, M. and Fatemi Ghomi, S. M. T. Simultaneously Solving of Balancing and Sequencing Problems with Station-dependent Assembly Times for Mixed-model Assembly Lines. Applied Soft Computing. 2012. 12: 1359-1370. 